\title{
QCD GLUEBALL SUM RULES AND VACUUM TOPOLOGY
}

\author{
HILMAR FORKEL* \\ Institute for Theoretical Physics, University of Heidelberg \\ D-69102 Heidelberg, Germany \\ and \\ IFT-UNESP \\ 01405-900 - São Paulo, SP, Brazil \\ *E-mail: forkel@ift.unesp.br
}

\begin{abstract}
Several key problems of QCD sum rules in the spin-0 glueball channels are resolved by implementing nonperturbative short-distance physics from direct instantons and topological charge screening. A lattice-based instanton size distribution and the IR renormalization of the nonperturbative Wilson coefficients are also introduced. Results of a comprehensive quantitative sum rule analysis are reviewed and their implications discussed.
\end{abstract}

\section{Introduction}

The gluonium states of QCD have remained intriguing for almost four decades ${ }^{1}$. Their "exotic" nature reflects itself not least in several longstanding problems which the QCD sum rule approach faces in the spin0 glueball channels ${ }^{2}$. In the scalar $\left(0^{++}\right)$glueball correlator, in particular, the departure from asymptotic freedom sets in at unusually small distances ${ }^{3}$ and the perturbative Wilson coefficients of the standard operator product expansion (OPE) proved inadequate to establish consistency both among the $0^{++}$glueball sum rules and with an underlying low-energy theorem ${ }^{4}$. Although nonperturbative contributions due to direct (i.e. small) instantons ${ }^{5}$ were early candidates for the missing short-distance physics ${ }^{6}$, insufficient knowledge of the instanton size distribution ${ }^{5,7}$ prevented their quantitative implementation at the time. Only recently, the derivation of the exact instanton contributions (to leading order in $\hbar$ ), their duality continua and the corresponding Borel sum-rule analysis ${ }^{4}$ showed that direct instantons indeed solve the mentioned key problems in the scalar glueball channel. Below we will outline a more thorough and systematic treatment ${ }^{9}$ which eliminates artefacts of earlier approximations, significantly modifies the sum-rule 
results in both spin- 0 channels and improves their reliability. Moreover, we implement for the first time topological charge screening contributions to the OPE in the $0^{-+}$channel $^{9}$ and show how those resolve earlier sumrule inconsistencies ${ }^{10}$. Finally, we review several new predictions for spin-0 glueball properties.

\section{Correlators and sum rules}

The spin- 0 glueball sum rules are based on the scalar $\left(0^{++}\right)$and pseudoscalar $\left(0^{-+}\right)$correlations functions

$$
\Pi_{G}\left(-q^{2}\right)=i \int d^{4} x e^{i q x}\left\langle 0\left|T O_{G}(x) O_{G}(0)\right| 0\right\rangle
$$

where $O_{G}$ with $G \in\{S, P\}$ are the gluonic interpolating fields (of lowest mass dimension) $O_{S}=\alpha_{s} G_{\mu \nu}^{a} G^{a \mu \nu}$ and $O_{P}=\alpha_{s} G_{\mu \nu}^{a} \tilde{G}^{a \mu \nu}$ with $\tilde{G}_{\mu \nu} \equiv(1 / 2) \varepsilon_{\mu \nu \rho \sigma} G_{\rho \sigma}$ (the definition in Ref. 9 contains a typo). The zeromomentum limit of the correlator (1) is governed by the low-energy theorems (LETs)

$$
\Pi_{S}\left(q^{2}=0\right)=\frac{32 \pi}{b_{0}}\left\langle\alpha G^{2}\right\rangle
$$

in the scalar ${ }^{11}$ and (for three light flavors and $m_{u, d} \ll m_{s}$ )

$$
\Pi_{P}\left(q^{2}=0\right)=(8 \pi)^{2} \frac{m_{u} m_{d}}{m_{u}+m_{d}}\langle\bar{q} q\rangle
$$

in the pseudoscalar channel ${ }^{12}$. (Note that Eq. (3) vanishes in the chiral limit.) Consistency with the low-energy theorems places stringent constraints on the sum rules which cannot be satisfied without nonperturbative short-distance physics ${ }^{4,9}$.

Contact with the hadronic information in the glueball correlators is established by means of the dispersive representation

$$
\Pi_{G}\left(Q^{2}\right)=\frac{1}{\pi} \int_{0}^{\infty} d s \frac{\operatorname{Im} \Pi_{G}(-s)}{s+Q^{2}}
$$

where the necessary number of subtractions is implied but not written explicitly. The standard sum-rule description of the spectral functions

$$
\operatorname{Im} \Pi_{G}^{(p h)}(s)=\operatorname{Im} \Pi_{G}^{(p o l e)}(s)+\operatorname{Im} \Pi_{G}^{(c o n t)}(s)
$$

contains one or two resonance poles in zero-width approximation, $\operatorname{Im} \Pi^{(\text {pole })}(s)=\pi \sum_{i=1}^{2} f_{G i}^{2} m_{G i}^{4} \delta\left(s-m_{G i}^{2}\right)$, and the local-duality continuum $\operatorname{Im} \Pi_{G}^{(\text {cont })}(s)=\theta\left(s-s_{0}\right) \operatorname{Im} \Pi_{G}^{(I O P E)}(s)$ from the IOPE discontinuities in the "duality range" which starts at an effective threshold $s_{0}$. 
In order to write down QCD sum rules, the Borel-transformed IOPE and dispersive representations of the correlators - weighted by $k$ powers of $-Q^{2}$ - are matched in the fiducial $\tau$-region ( $\tau$ is the Borel variable) and rearranged in terms of the continuum-subtracted Borel moments $\mathcal{R}_{G, k}$ as

$$
\begin{aligned}
\mathcal{R}_{G, k}\left(\tau ; s_{0}\right) & \equiv \frac{1}{\pi} \int_{0}^{s_{0}} d s s^{k} \operatorname{Im} \Pi_{G}^{(I O P E)}(s) e^{-s \tau} \\
& =\sum_{i=1}^{2} f_{G i}^{2} m_{G i}^{4+2 k} e^{-m_{G i}^{2} \tau}-\delta_{k,-1} \Pi_{G}^{(p h)}(0) .
\end{aligned}
$$

The pole contributions of interest (and for $k=-1$ the crucial subtraction terms) are then isolated on the RHS, and the hadronic parameters $m_{G i}, f_{G i}$ and $s_{0}$ can be determined numerically.

\section{IOPE}

Our theoretical framework, the instanton-improved operator product expansion (IOPE), factorizes the correlators at large, spacelike momenta $Q^{2} \equiv-q^{2} \gg \Lambda_{Q C D}$ into contributions from "hard" field modes (with momenta $|k|>\mu)$ in the Wilson coefficients $\tilde{C}_{D}\left(Q^{2}\right)$ and "soft" field modes (with $|k| \leq \mu$ ) in the "condensates" $\left\langle\hat{O}_{D}\right\rangle_{\mu}$ of operators $\hat{O}_{D}$ with increasing dimension $D$. Previous glueball sum rules based on the OPE with purely perturbative Wilson coefficients were plagued by notorious inconsistencies between the predictions of different moment sum rules and by massive LET violations. Moreover, the soft nonperturbative condensate contributions were exceptionally small.

We have therefore analyzed hard nonperturbative contributions to the Wilson coefficients. They are strongly channel dependent and due to direct instantons and topological charge screening. The instanton contributions to the spin-0 coefficients ${ }^{9}$,

$$
\Pi_{G}^{(I+\bar{I})}\left(x^{2}\right)=\frac{2^{8} 3}{7} \int d \rho n(\rho) \frac{1}{\rho^{4}}{ }_{2} F_{1}\left(4,6, \frac{9}{2},-\frac{x^{2}}{4 \rho^{2}}\right),
$$

are large (while those in the $2^{++}$tensor channel vanish) and add to the unitoperator coefficients $\tilde{C}_{0}^{(G)}$. The imginary part of their Fourier transform at timelike momenta generates the Borel moments ${ }^{4}$

$$
\begin{aligned}
\mathcal{R}_{k}^{(I+\bar{I})}(\tau) & =-2^{7} \pi^{2} \delta_{k,-1} \int d \rho n(\rho)-2^{4} \pi^{3} \int d \rho \\
& \times n(\rho) \rho^{4} \int_{0}^{s_{0}} d s s^{k+2} J_{2}(\sqrt{s} \rho) Y_{2}(\sqrt{s} \rho) e^{-s \tau}
\end{aligned}
$$


which are similar or larger in size than the perturbative ones. The evaluation of these moments requires as the sole input the (anti-) instanton distribution $n(\rho)$ which is implemented by means of a lattice-based Gaussian-tail parametrization with the correct small- $\rho$ behavior,

$$
n_{g}(\rho)=\frac{2^{18}}{3^{6} \pi^{3}} \frac{\bar{n}}{\bar{\rho}}\left(\frac{\rho}{\bar{\rho}}\right)^{4} \exp \left(-\frac{2^{6}}{3^{2} \pi} \frac{\rho^{2}}{\bar{\rho}^{2}}\right)
$$

(for $N_{c}=N_{f}=3$ ), which was introduced in Ref. 9 and shown to prevent several artefacts of the oversimplified "spike" approximation $n(\rho)=$ $\bar{n} \delta(\rho-\bar{\rho})$ on which all previous direct-instanton calculations had relied.

As an additional benefit, the realistic size distribution allows for a gaugeinvariant IR renormalization which excludes large instantons with size $\rho>$ $\mu^{-1}$ from the Wilson coefficients,

$$
n(\rho) \rightarrow \tilde{n}(\mu ; \rho) \equiv \theta_{\beta}\left(\rho-\mu^{-1}\right) n(\rho)
$$

( $\theta_{\beta}$ is a soft step function). The instanton-induced $\mu$ dependence turns out to be relatively weak for $\mu<\bar{\rho}^{-1}$, as necessary to compensate its perturbative counterpart. Neglect of this renormalization, although common practice in perturbative Wilson coefficients, would significantly contaminate the results, e.g. by missing the reduction of the direct-instanton density

$$
\bar{n}=\int_{0}^{\infty} d \rho n(\rho) \rightarrow \int_{0}^{\infty} d \rho \tilde{n}(\mu ; \rho) \equiv \bar{n}(\mu) .
$$

Another important renormalization effect is the reduction of the instanton contributions to the pseudoscalar relative to the scalar sum rules.

The instanton's self-duality causes a strongly repulsive contribution to the $0^{-+}$channel, with seemingly detrimental impact on the sum rules ${ }^{10}$ : the glueball signal disappears and both unitarity and the LET (3) are badly violated. The origin of these problems can be traced to the neglect of topological charge screening ${ }^{9}$. Due to their high channel selectivity and a small screening length $\lambda_{D} \sim m_{\eta^{\prime}}^{-1} \sim 0.2 \mathrm{fm}$, the model-independent screening correlations $^{14}$

$$
\Pi_{P}^{(s c r)}(x) \simeq-2^{8} \pi^{2}\left(\xi \gamma_{\eta_{0}}\right)^{2}\left\langle\eta_{0}(x) \eta_{0}(0)\right\rangle
$$

( $\xi$ is the overall topological charge density ( $=\bar{n}$ for instantons) and $\eta_{0}$ the flavor-singlet part of the $\eta^{\prime}$ ) affect almost exclusively the $0^{-+}$Wilson coefficients. They arise from the axial-anomaly induced attractive (repulsive) interaction between topological charge lumps of opposite (equal) sign due to $\eta_{0}$ exchange $^{13}$ and, after correcting for $\eta_{0}-\eta_{8}$ mixing, add the terms

$$
\mathcal{R}_{P, k}^{(s c r)}(\tau)=-\delta_{k,-1}\left(\frac{F_{\eta^{\prime}}^{2}}{m_{\eta^{\prime}}^{2}}+\frac{F_{\eta}^{2}}{m_{\eta}^{2}}\right)+F_{\eta^{\prime}}^{2} m_{\eta^{\prime}}^{2 k} e^{-m_{\eta^{\prime}}^{2} \tau}+F_{\eta}^{2} m_{\eta}^{2 k} e^{-m_{\eta}^{2} \tau}
$$


to the pseudoscalar IOPE moments.

They strongly reduce the direct-instanton induced repulsion and resolve the disastrous problems mentioned above ${ }^{9}$ : positivity of the spectral function is restored, the four $0^{-+}$Borel sum rules (6) are stable and contain consistent pseudoscalar glueball information. (All previous analyses had discarded the $k=-1$ sum rule and thereby missed valuable first-principle information and LET consistency checks.)

\section{Results and discussion}

We have implemented direct instanton and topological charge screening contributions into the OPE coefficients of the spin-0 glueball correlators, evaluated their duality continuua and demonstrated how these contributions resolve the problems of previous QCD sum rule analyses ${ }^{9}$. A latticebased instanton size distribution and the gauge-invariant IR renormalization of the nonperturbative Wilson coefficients were also introduced. Quark admixtures, and thereby quarkonium mixing effects, enter through quark loops, the instanton size distribution and the condensates.

In the scalar channel, the sizeable direct instanton contributions are indispensable for mutually and LET consistent sum rules. Their improved treatment reduces our earlier (spike-distribution based) result for the $0^{++}$ glueball mass to $m_{S}=1.25 \pm 0.2 \mathrm{GeV}$. (The mass stays well beyond 1 $\mathrm{GeV}$, however, in contrast to obsolete predictions based on purely perturbative coefficients.) This value is somewhat smaller than the quenched lattice results $^{15}$ (which will probably be reduced by light-quark effects) and consistent with the broad glueball state found in a comprehensive $K$-matrix analysis ${ }^{16}$. The systematics among our different Borel moments likewise indicates a rather large width of the scalar glueball, $\Gamma_{S} \gtrsim 0.3 \mathrm{GeV}$.

Our prediction for the scalar glueball decay constant, $f_{S}=1.05 \pm 0.1$ $\mathrm{GeV}$, is several times larger than the value obtained when ignoring the nonperturbative Wilson coefficients. This implies an exceptionally small $0^{++}$ glueball size, in agreement with several lattice results ${ }^{17}$. Another stringent, OPE- and sum-rule-independent consistency check of the instanton contributions and their $f_{S}$ enhancement provide numerical simulations in an instanton ensemble ${ }^{18}$ (ILM). For $|x| \lesssim 0.5 \mathrm{fm}$ the scalar ILM and IOPE correlators are indeed very similar, and the ILM prediction $f_{S}^{(I L M)}=0.8$ $\mathrm{GeV}$ is similarly large. This indicates a robust instanton effect and rules out that the large $f_{S}$ "may signal some eventual internal inconsistencies in the treatment of the instanton contributions"19. A subsequent modelindependent confirmation of the $f_{S}$ enhancement was supplied by the first 
direct (quenched) lattice result ${ }^{20} f_{S}=0.86 \pm 0.18 \mathrm{GeV}$ which is consistent both with our prediction and the ILM value (the latter is practically independent of quark quenching ${ }^{18}$ ).

Our prediction for $f_{S}$ implies enhanced partial widths for radiative $J / \psi$ and $\Upsilon$ decays into scalar glueballs and is therefore relevant for experimental glueball searches, e.g. in the CLEO and BES data on $\Upsilon \rightarrow \gamma f_{0}$ and other decay branches. Since the exceptionally small size and large decay constant are particular to the scalar glueball and since only part of the scalar decay constant contributes to the radiative production rates ${ }^{21}$, however, the above results are not ruled out by experimental data on decays into $0^{-+}$glueballs (cf. Ref. 19).

In the pseudoscalar $\left(0^{-+}\right)$glueball correlator we have identified and implemented a new type of nonperturbative contributions to the Wilson coefficients, due to topological charge screening. Roughly speaking, the screening effects "unquench" the direct instanton contributions, thereby restoring unitarity, the axial Ward identity and the resonance signals. Consistency among all moment sum rules and with the underlying LET is also achieved, and the resulting mass prediction $m_{P}=2.2 \pm 0.2 \mathrm{GeV}$ lies inside the range of quenched and unquenched lattice data. The coupling $f_{P}=0.6 \pm 0.25$ $\mathrm{GeV}$ is somewhat enhanced by the topological short-distance physics, affecting radiative production rates, the $\gamma \gamma \rightarrow G_{P} \pi^{0}$ cross section at high momentum transfers and other glueball signatures.

The crucial impact of the nonperturbative Wilson coefficients on both spin-0 glueball correlators is particularly evident in the interplay between their subtraction constants. Indeed, the notorious consistency problems which plagued previous $0^{++}$glueball sum rules were primarily caused by the large LET-induced subtraction constant $\Pi_{S}(0) \simeq 0.6 \mathrm{GeV}^{4}$ (cf. Eq. (2)): it cannot be matched by perturbative Wilson coefficients (since $\Pi_{S}^{(\text {pert })}(0)=$ 0 ) and requires the direct instanton contribution $\Pi_{S / P}^{(I, \bar{I})}(0)= \pm 2^{7} \pi^{2} \bar{n}_{\text {dir }} \simeq$ $0.63 \mathrm{GeV}^{4}$. At first sight this seems to imply a conflict with the much smaller LET subtraction constant $\Pi_{P}(0) \simeq-0.02 \mathrm{GeV}^{4}$ (cf. Eq. (3)) in the $0^{+-}$channel, however, since the instanton contributions to both spin- 0 correlators are equal (up to a sign). Here the topological screening contributions $\Pi_{P}^{(s c r)}(0) \simeq 0.59 \mathrm{GeV}^{4}$ from Eq. (13) prove indispensable: they restore consistency by canceling most (and in the chiral limit, where $\Pi_{P}(0) \rightarrow 0$, all) of the instanton contributions: $\Pi_{P}^{(s c r)}(0)+\Pi_{P}^{(I, \bar{I})}(0) \simeq \Pi_{P}(0)$.

To summarize: contrary to naive expectation, the nonperturbative contributions to the OPE of the spin-0 glueball correlators reside primarily in 
the Wilson coefficients (i.e not in the condensates) and are closely related to the topological vacuum structure. The nonperturbative short-distance physics resolves long-standing consistency problems of the associated QCD sum rules and generates a rather diverse set of new glueball predictions. A large part of the $0^{++}$glueball mass and binding originates from direct instantons, for example, while their net effects in the $0^{+-}$channel are smaller

and more subtle, due to cancellations between instanton and topological charge screening contributions.

This work was supported by FAPESP and CNPq of Brazil.

\section{References}

1. M. Gell-Mann, Acta Phys. Aust. Suppl. 9, 733 (1972); H. Fritzsch and M. Gell-Mann, 16th Int. Conf. High-Energy Phys., Chicago, Vol. 2, 135 (1972).

2. S. Narison, Nucl. Phys. B509, 312 (1998) and references therein.

3. V.A. Novikov, M.A. Shifman, A.I. Vainsthein, and V.I. Zakharov, Nucl. Phys. B191, 301 (1981).

4. H. Forkel, Phys. Rev. D 64, 034015 (2001).

5. T. Schäfer and E.V. Shuryak, Rev. Mod. Phys. 70, 323 (1998). For an introduction see H. Forkel, A Primer on Instantons in QCD, hep-ph/0009136.

6. V.A. Novikov, M.A. Shifman, A.I. Vainsthein, and V.I. Zakharov, Nucl. Phys. B165, 67 (1980).

7. A. Ringwald and F. Schremmp, Phys. Lett. B 459, 249 (1999).

8. D. Harnett and T.G. Steele, Nucl. Phys. A 695, 205 (2001).

9. H. Forkel, Phys. Rev. D 71, 054008 (2005); Braz. J. Phys. 34, 875 (2004); AIP Conf. Proc. 739, 434 (2004).

10. A. Zhang and T.G. Steele, Nucl. Phys. A 728, 165 (2003).

11. V.A. Novikov, M.A. Shifman, A.I. Vainshtein and V.I. Zakharov, Nucl. Phys. B191, 301 (1981).

12. H. Leutwyler and A. Smilga, Phys. Rev. D 46, 5607 (1992).

13. P. Di Vecchia and G. Veneziano, Nucl. Phys. B 171, 253 (1980).

14. N.J. Dowrick and N.A. McDougall, Phys. Lett. B 285, 269 (1992); H. Kikuchi and J. Wudka, Phys. Lett. B 284, 111 (1992).

15. W. Lee and D. Weingarten, Phys. Rev. D 61, 014015 (2000) and references therein; C. Morningstar and M. Peardon, Phys. Rev. D 60, 034509 (1999).

16. V.V. Anisovich, AIP Conf. Proc. 717, 441 (2004), arXiv:hep-ph/0310165.

17. N. Ishii, H. Suganuma, and H. Matsufuru, Phys. Rev. D 66, 94506 (2002); P. de Forcrand and K.-F. Liu, Phys. Rev. Lett. 69, 245 (1992); R. Gupta et al., Phys. Rev. D 43, 2301 (1991).

18. T. Schäfer and E.V. Shuryak, Phys. Rev. Lett. 75, 1707 (1995).

19. S. Narison, arXiv:hep-ph/0512256.

20. Y. Chen et al., Phys. Rev. D 73, 014516 (2006).

21. X.-G. He, H.-Y. Jin and J. P. Ma, Phys. Rev. D 66, 74015 (2002). 